\title{
Interactive comment on "Multi-year Evaluation of Airborne Geodetic Surveys to Estimate Seasonal Mass Balance, Columbia and Rocky Mountains, Canada" by Ben M. Pelto et al.
}

\section{Anonymous Referee \#2}

Received and published: 26 March 2019

Pelto and colleagues present a detailed evaluation of airborne geodetic datasets, obtained between 2014 and 2018, to estimate seasonal mass balances on 6 glaciers in the Columbia and Rocky Mountains. This study puts airborne laser scanner (ALS) dataset with exceptional spatial and temporal resolution at its full value. The comparison of these geodetic results with the glaciological balances from in-situ measurements are carried out in a very thorough way, including an error assessment according to best international practises. I found this paper to be well thought out and mostly well described. However, in some cases the writing was a bit unclear (see comments below). The results from this work do contribute scientifically to methods and knowledge pertaining to mass balance and I would recommend the paper to be considered for

Printer-friendly version

Discussion paper 
publication after consideration of the following points and a list of suggestions for minor corrections and clarifications:

DTM-related uncertainties:

Which interpolation algorithms have been used for DTM production? Please state in one paragraph!

The authors use the mean, median and normalized median absolute deviation (NMAD) of the DTM-differencing over selected stable terrain as "systematic" uncertainty (Bias $\Delta h$ ). It is unclear through the manuscript, how overall hdDEM was determined? Please describe in more detail! Furthermore, is hdDEM the same as Bias $\Delta h$ ? If so, please streamline through the manuscript!

The authors have stated not to use their gridded data for correction of their sample sizes for spatial autocorrelation (L103-104). Please state what was used instead (point cloud data?) and, if so, have the point cloud datasets also been co-registered beforehand? To calculate uncertainty in ALS-derived height change, the authors account for spatial correlation, assessed over stable terrain based on semi-variogram analysis as described in Rolstad et al. (2009). How did the authors determine $\sigma$ h and what are the values of $\sigma$ hdDEM Please describe in more detail and present the values in a Table (also possible in Supplement).

\section{Specific comments}

L19: delete aiborne, redundant to ALS

L26ff: I recommend to align the references based on the date of appearance. Streamline through the manuscript.

L28: "Measurement of seasonal mass change provides..." , you mean in situ measurements or do you refer to all methods?

L35: Please rephrase sentence! 
L37-41: This paragraph could be improved, giving a bit more substance!

L51: abbreviation CBR has to be introduced earlier

L68-71: Please indicate the extent of the major mountain ranges in Figure 1.

L69: try to omit redundant information, which improves readability: eg. rephrase sentence to (1) Zillmer Glacier (5.4 km2) in the Cariboo Mountains, (2) Nordic Glacier (3.4 km2) and (3) Illecillewaet Glacier (7.7 km2) in the Selkirk Mountains, (4) Conrad Glacier $(11.5 \mathrm{~km} 2)$ and (5) Kokanee Glacier (1.8 km2) in the Purcell Mountains, and (6) Haig Glacier (2.6 km2), which straddles the continental divide in the Rocky Mountains.

L77: swatch change to swath

L82: Please state in one paragraph, which interpolation algorithms you used!

L117: It would be helpful to describe what 'snow course' data is.

L256: 'glacier-wise' is an unclear term, but used quite often throughout the manuscript. Is it possible to change to another more intuitive term?

L281: omit hyphen in lower-accumulation

L282: Which unit does 0.06 have? Think $\mathrm{km}^{2}$ ? I thought Haig glacier is not in the Columbia Mountains?

L331: $\sigma$ hdDEM is not in Table 3, see comment above

L373: 'In western Canada, onset of snow melt is occurring earlier on average relative to $1970-2006$ '. For what period is the onset of earlier snow melt occurring? Please give detrails.

L407-411: 'Our field operations have been impacted by the melting out of crevasses: as strongly negative years are becoming the norm, and glacier flux is likely decreasing, crevasses are exposed for longer periods of time, and slower to close. This means that the total void area of crevasses is increasing due to ablation, which we have ob- 
served on Conrad, Zillmer, Nordic, and Haig glaciers, which could possibly increase their influence on Bw.'

This part is a bit unclear! What is meant with melting out of crevasses, please clarify. Which flux decreases (surface velocity or mass balance or both)? Give references of the source your assumption is based on! How was the increase of the void area of crevasses due to ablation observed? Can you detail this?

L426: The statement "Our glaciological measurement densities ranged from 0.5 to 18.5 points km-2 (Table2), whereas our ALS data had around one million points km-2" is a bit of comparing pears with apples. Please discuss in more detail or omit!

Figures: text is of variable font and size within figures. Especially on figures 4 and 7 the text is hardly readable

Interactive comment on The Cryosphere Discuss., https://doi.org/10.5194/tc-2019-30, 2019. 